\title{
THE BOUNDARY OF THE KREIN SPACE TRACIAL NUMERICAL RANGE, AN ALGEBRAIC APPROACH AND A NUMERICAL ALGORITHM
}

\author{
N. BEBIANO, H. NAKAZATO, A. NATA AND J. DA PROVIDÊNCIA
}

\begin{abstract}
In this article, tracial numerical ranges associated with matrices in an indefinite inner product space are investigated. The boundary equations of these sets are obtained and the case of the boundary being a polygon is studied. As an application, a numerical algorithm for plotting the tracial numerical range of an arbitrary complex matrix, is presented. Our approach uses the elementary idea that the boundary may be traced by computing the supporting lines.
\end{abstract}

KEYWORDS: Numerical range, indefinite inner product, $J$-Hermitian matrix, noninterlacing eigenvalues.

AMS Subject Classification (2000): 47A12, 46C20.

\section{Introduction}

Let $J$ be a Hermitian involutive matrix (i.e., $J^{*}=J, J^{2}=I_{n}$ ), with signature $(r, n-r), 0 \leq r \leq n$ (i.e., with $r$ positive and $n-r$ negative eigenvalues). We consider $\mathbb{C}^{n}$ endowed with the indefinite inner product defined by $[x, y]=y^{*} J x, x, y \in \mathbb{C}^{n}$. A matrix $H \in M_{n}$, the algebra of $n \times n$ complex matrices, is $J$-Hermitian if $H^{\#}=H$, where $H^{\#}=J H^{*} J$ is the $J$ adjoint of $H$. A matrix $U \in M_{n}$ is said to be $J$-unitary if $U^{\#} U=U U^{\#}=I_{n}$. The $J$-unitary matrices form a locally compact connected group denoted by $\mathcal{U}_{r, n-r}$ and called the J-unitary group. In the case $r=n, \mathcal{U}_{n, 0}=\mathcal{U}_{n}$ is the unitary group, which is a compact group. For $C, A \in M_{n}$, the J-tracial numerical range of $A$ is denoted and defined as

$$
W_{C}^{J}(A)=\left\{\operatorname{tr}\left(\mathrm{CUAU}^{-1}\right): \mathrm{U} \in \mathcal{U}_{\mathrm{r}, \mathrm{n}-\mathrm{r}}\right\} .
$$

This set is a connected set in the Argand plane $\mathbb{C}$ and satisfies the symmetry property $W_{C}^{J}(A)=W_{A}^{J}(C)$ (see [1], [6] for other properties). If $r=n, W_{C}^{J}(A)$ is the $C$-numerical range of $A$ simply denoted by $W_{C}(A)$. Hausdorff [3] proved the convexity of $W_{C}(A)=W(A)$ in the case $C$ is a rank one orthogonal projection. Using Morse theory, Westwick [10] proved the convexity of $W_{C}(A)$

Received October 1, 2009. 
for $C$ Hermitian, and in [8] Poon gave an alternative proof using majorization techniques. In [9], Tam generalized Westwick's theorem for certain compact Lie groups and recently [7], a convexity result for $W_{C}^{J}(A)$ has been proved applying Morse theory.

We recall that the spectrum of a $J$-Hermitian matrix is symmetric with respect to the real axis. In this note, to avoid trivial cases we mainly consider $J$-Hermitian matrices with real spectra. In the sequel the following notation will be used:

$$
\begin{aligned}
& \sigma_{+}^{J}(A)=\left\{\lambda \in \mathbb{C}: A \xi=\lambda \xi, \text { for some } \xi \in \mathbb{C}^{n},[\xi, \xi]>0\right\}, \\
& \sigma_{-}^{J}(A)=\left\{\lambda \in \mathbb{C}: A \xi=\lambda \xi, \text { for some } \xi \in \mathbb{C}^{n},[\xi, \xi]<0\right\} .
\end{aligned}
$$

The eigenvalues of $A$ are said to be noninterlacing if

$$
\max \sigma_{-}^{J}(A)<\min \sigma_{+}^{J}(A) \quad \text { or } \max \sigma_{+}^{J}(A)<\min \sigma_{-}^{J}(A) .
$$

Replacing in $(4)<$ by $\leq$ the eigenvalues are said to be weakly noninterlacing. Noninterlacing property plays a key role in the development of the theory of numerical ranges associated with non-compact groups.

Let $\Re^{J}(A)=\left(A+A^{\#}\right) / 2$ and $\Im^{J}(A)=\left(A-A^{\#}\right) / 2 i$ be the unique $J$-Hermitian matrices such that $A=\Re^{J}(A)+i \Im^{J}(A)$. If $J=I_{n}$ this reduces to the well-known cartesian decomposition of $A, A=\Re(A)+i \Im(A)$, where $\Re(A)=\left(A+A^{*}\right) / 2$ and $\Im(A)=\left(A-A^{*}\right) / 2 i$. Throughout, $C$ will denote a $J$-Hermitian matrix with noninterlacing eigenvalues:

$$
\begin{gathered}
\sigma_{+}^{J}(C)=\left\{c_{1} \geq \cdots \geq c_{r}\right\}, \quad \sigma_{-}^{J}(C)=\left\{c_{r+1} \geq \cdots \geq c_{n}\right\}, \\
c_{n}>c_{1} \text { or } c_{r}>c_{r+1} .
\end{gathered}
$$

Under these hypothesis $C$ is $J$-unitarily diagonalizable, that is, diagonalizable under a $J$-unitary similarity transformation. In [7] it has been proved that if there exists $\theta \in\left[\theta_{1}, \theta_{2}\right] \subset \mathbb{R}$ such that $H_{\theta}:=\Re^{J}\left(e^{-i \theta} A\right)$ has real noninterlacing eigenvalues

$$
\begin{gathered}
\sigma_{+}^{J}\left(H_{\theta}\right)=\left\{\lambda_{1}(\theta) \geq \cdots \geq \lambda_{r}(\theta)\right\}, \quad \sigma_{-}^{J}\left(H_{\theta}\right)=\left\{\lambda_{r+1}(\theta) \geq \cdots \geq \lambda_{n}(\theta)\right\}, \\
\lambda_{n}(\theta)>\lambda_{1}(\theta) \text { or } \lambda_{r}(\theta)>\lambda_{r+1}(\theta),
\end{gathered}
$$

then $W_{C}^{J}(A)$ is a closed convex set in $\mathbb{C}$ contained in a closed cone

$$
\left\{z_{0}+r e^{i \eta}: r \geq 0, \eta_{1} \leq \eta \leq \eta_{2}\right\}
$$

with $0 \leq \eta_{2}-\eta_{1}<\pi$. 
This paper is organized as follows. The boundary equations of $W_{C}^{J}(A)$ are deduced in Section 2. In Section $3, W_{C}^{J}(A)$ is studied for $A$ a $J$-normal matrix. As an heuristic tool, it is convenient to have a code to produce the plot of $W_{C}^{J}(A)$. In Section 4 an algorithm to plot $W_{C}^{J}(A)$ is presented. Our approach uses the elementary idea that the boundary of $W_{C}^{J}(A)$ may be traced by computing the supporting lines of $W_{C}^{J}(A)$.

\section{On the boundary of $W_{C}^{J}(A)$}

Theorem 2.1. Let $J=I_{r} \oplus-I_{n-r}, 0<r<n, C=\operatorname{diag}\left(c_{1}, \cdots, c_{n}\right)$ with $c_{1}>\cdots>c_{r} \in \sigma_{+}^{J}(C), c_{r+1}>\cdots>c_{n} \in \sigma_{-}^{J}(C)$ and $c_{n}>c_{1}$. Let $A \in M_{n}$ be a non $J$-normal matrix. Assume that there exists $\theta \in\left[\theta_{1}, \theta_{2}\right]$ such that the $J$-Hermitian matrix $\Re^{J}\left(\mathrm{e}^{-i \theta} A\right)$, has real simple and noninterlacing eigenvalues:

$$
\lambda_{1}(\theta)>\cdots>\lambda_{r}(\theta)>\lambda_{r+1}(\theta)>\cdots>\lambda_{n}(\theta) .
$$

Then the function $\Lambda(\theta)=\sum_{j=1}^{n} c_{j} \lambda_{j}(\theta)$ is convex in the domain

$$
\left\{r \mathrm{e}^{i \theta}: r>0, \theta_{1}<\theta<\theta_{2}\right\}
$$

and the curve parametrized by

$$
\left\{\begin{array}{l}
x(\theta)=\cos \theta \Lambda(\theta)-\sin \theta \Lambda^{\prime}(\theta) \\
y(\theta)=\sin \theta \Lambda(\theta)+\cos \theta \Lambda^{\prime}(\theta)
\end{array}, \quad \theta \in\left[\theta_{1}, \theta_{2}\right]\right.
$$

is contained in $\partial W_{C}^{J}(A)$.

Proof. Let $A=H+i K$ be a non $J$-normal matrix, where $H=\Re^{J}(A)$ and $K=\Im^{J}(A)$. Under the hypothesis, the $J$-Hermitian matrix $\Re^{J}\left(\mathrm{e}^{-i \theta} A\right)=$ $\cos \theta H+\sin \theta K$ has real simple eigenvalues for $\theta \in\left[\theta_{1}, \theta_{2}\right]$. Then there exists a $J$-orthonormal basis in $\mathbb{C}^{n},\left\{\xi_{1}(\theta), \cdots, \xi_{n}(\theta)\right\}$, satisfying $\Re^{J}\left(\mathrm{e}^{-i \theta} A\right) \xi_{j}(\theta)=$ $\lambda_{j}(\theta) \xi_{j}(\theta), j=1, \cdots, n$. According to the hypothesis and by Theorem 1.1 of [1]

$$
W_{C}^{J}\left(\Re^{J}\left(\mathrm{e}^{-i \theta} A\right)\right)=\left(-\infty, \sum_{j=1}^{n} c_{j} \lambda_{j}(\theta)\right] .
$$

The eigenvalue $\lambda_{j}(\theta)$ is an analytic function of $\theta$. Since the eigenvalues are simple, we may assume that $\xi_{j}(\theta)$ is an analytic function of $\theta$. The image of 
the map $\phi:\left[\theta_{1}, \theta_{2}\right] \rightarrow \mathbb{C}$ defined by

$$
\begin{aligned}
\phi(\theta) & =u(\theta)+i v(\theta) \\
& =\sum_{j=1}^{n} c_{j} \mathrm{e}^{i \theta}\left[\frac{\left[\left(\Re^{J}\left(\mathrm{e}^{-i \theta} A\right) \xi_{j}(\theta), \xi_{j}(\theta)\right]\right.}{\left[\xi_{j}(\theta), \xi_{j}(\theta)\right]}+i \frac{\left[\left(\Im^{J}\left(\mathrm{e}^{-i \theta} A\right) \xi_{j}(\theta), \xi_{j}(\theta)\right]\right.}{\left[\xi_{j}(\theta), \xi_{j}(\theta)\right]}\right]
\end{aligned}
$$

is contained in the boundary of $W_{C}^{J}(A)$. The dual curve of the curve $\phi(\theta)$, that is, the family of tangent lines of the curve $\phi(\theta)$, may be defined in an interval $\left[\theta_{1}, \theta_{2}\right]$ on which $\phi^{\prime}(\theta) \neq 0$. The dual curve is given by

$$
\left\{\left(1, \frac{-v^{\prime}(\theta)}{u(\theta) v^{\prime}(\theta)-v(\theta) u^{\prime}(\theta)}, \frac{u^{\prime}(\theta)}{u(\theta) v^{\prime}(\theta)-v(\theta) u^{\prime}(\theta)}\right): \theta_{1}<\theta<\theta_{2}\right\} .
$$

The homogeneous expression of the dual curve $L$ is given by

$$
\left\{\left(\sum_{j=1}^{n} c_{j} \lambda_{j}(x H+y K),-x,-y\right):(x, y) \in \mathbb{R}^{2}\right\} .
$$

In an nonhomogeneous form, the dual curve is given by

$$
\tilde{L}=\left\{\left(-x^{-1} \sum_{j=1}^{n} c_{j} \lambda_{j}(x H+y K), 1, \frac{y}{x}\right): \arctan \theta_{1}<\frac{y}{x}<\arctan \theta_{2}\right\},
$$

or by

$$
L=\left\{\left(1, \frac{-\cos \theta}{\sum_{j=1}^{n} c_{j} \lambda_{j}(\theta)}, \frac{-\sin \theta}{\left.\sum_{j=1}^{n} c_{j} \lambda_{j}(\theta)\right)}\right): \theta_{1}<\theta<\theta_{2}\right\} .
$$

Next we show that the function

$$
f(x, y)=x f\left(1, \frac{y}{x}\right)=\sum_{j=1}^{n} c_{j} \lambda_{j}(x H+y K)
$$

is convex in the domain $\left\{r \mathrm{e}^{i \theta}: r>0, \theta_{1}<\theta<\theta_{2}\right\}$.

For simplicity we denote $f(1, y / x)$ by $f(y / x)$, and so the proof of the convexity of the positive homogeneous function $f(x, y)$ reduces to the proof of the convexity of $f(y / x)$.

Suppose that $J=I_{r} \oplus-I_{n-r}$ and let $H=\operatorname{diag}\left(\lambda_{1}, \ldots, \lambda_{r}, \lambda_{r+1}, \ldots, \lambda_{n}\right)$ with $\lambda_{1}>\lambda_{2}>\cdots>\lambda_{r}>\lambda_{r+1}>\cdots>\lambda_{n}$ being $K=\left(\mu_{t j}\right)$. We set 
$\mu_{t t}=\mu_{t}$. Then we have

$$
\lambda_{t}(H+y K)=\lambda_{t}+y \mu_{t}+y^{2} \sum_{1 \leq j \leq r, j \neq t} \frac{-\left|\mu_{t j}\right|^{2}}{\lambda_{j}-\lambda_{t}}+y^{2} \sum_{r+1 \leq j \leq n} \frac{\left|\mu_{t j}\right|^{2}}{\lambda_{j}-\lambda_{t}}+O\left(y^{3}\right),
$$

for $t=1,2, \ldots, r$ and

$$
\lambda_{t}(H+y K)=\lambda_{t}+y \mu_{t}+y^{2} \sum_{1 \leq j \leq r} \frac{\left|\mu_{t j}\right|^{2}}{\lambda_{j}-\lambda_{t}}+y^{2} \sum_{r+1 \leq j \leq n, j \neq t} \frac{-\left|\mu_{t j}\right|^{2}}{\lambda_{j}-\lambda_{t}}+O\left(y^{3}\right),
$$

for $t=r+1, r+2, \ldots, n$. Hence we obtain

$$
\begin{aligned}
f(y) & =\sum_{t=1}^{n} c_{t} \lambda_{t}(H+y K)=\sum_{t=1}^{n} c_{t} \lambda_{t}+y \sum_{t=1}^{n} c_{t} \mu_{t}+y^{2} \sum_{1 \leq t<j \leq r} \frac{c_{t}-c_{j}}{\lambda_{t}-\lambda_{j}}\left|\mu_{t j}\right|^{2} \\
& +y^{2} \sum_{r+1 \leq t<j \leq n} \frac{c_{t}-c_{j}}{\lambda_{t}-\lambda_{j}}\left|\mu_{t j}\right|^{2}+y^{2} \sum_{r+1 \leq t \leq n, 1 \leq j \leq r} \frac{c_{j}-c_{t}}{\lambda_{t}-\lambda_{j}}\left|\mu_{t j}\right|^{2}+O\left(y^{3}\right)
\end{aligned}
$$

and so the function $f(y)$ satisfies

$$
\begin{aligned}
\frac{1}{2} f^{\prime \prime}(0) & =\sum_{1 \leq t<j \leq r} \frac{c_{t}-c_{j}}{\lambda_{t}-\lambda_{j}}\left|\mu_{t j}\right|^{2}+\sum_{r+1 \leq t<j \leq n} \frac{c_{t}-c_{j}}{\lambda_{t}-\lambda_{j}}\left|\mu_{t j}\right|^{2} \\
& +\sum_{r+1 \leq t \leq n, 1 \leq j \leq r} \frac{c_{j}-c_{t}}{\lambda_{t}-\lambda_{j}}\left|\mu_{t j}\right|^{2} \geq 0 .
\end{aligned}
$$

Thus, the function $f(y)$ is locally convex at the origin. Since every point $(x, y) \neq(0,0)$ can be rotated to a point $\left(x_{0}, 0\right)$ we may conclude that the function $\sum_{j=1}^{n} c_{j} \lambda_{j}(x H+y K)$ is convex in the mentioned domain and the above curve $\tilde{L}$ does not have flexes.

Remark 2.1. (i) The curve $\left\{x(\theta)+i y(\theta): \theta \in\left[\theta_{1}, \theta_{2}\right]\right\}$ in Theorem 2.1 is called the boundary generating curve of $W_{C}^{J}(A)$.

(ii) The assumption of $\Re^{J}\left(\mathrm{e}^{-i \theta} A\right)$ having noninterlacing eigenvalues implies that either $\lambda_{r}(\theta)>\lambda_{r+1}(\theta)$ or $\lambda_{n}(\theta)>\lambda_{1}(\theta)$. By an appropriate choice of $\theta$ (i.e., substituting $\theta$ by $\theta+\pi$ ), we can reduce the second alternative to the first one and so without loss of generality we treat only one of them.

Theorem 2.2. Let $A \in M_{n}$, and let $J, C$ be under the conditions of Theorem 2.1. If the eigenvalues of $C$ are all distinct and there exists at least one corner in $W_{C}^{J}(A)$, then $A$ is $J$-normal. 
Proof. For any real $t$ and $S$ a $J$-Hermitian matrix, $\exp (i t S)=I+i t S+$ $\theta\left(t^{2}\right)$ is $J$-unitary. Let $z=\operatorname{tr}(\mathrm{AC})$ be a corner. It can be easily seen that $\operatorname{tr}(\mathrm{S}[\mathrm{C}, \mathrm{A}])=0$. For $A=H+i K$, and $\theta \in\left[\theta_{1}, \theta_{2}\right]$, we have $\left[C, \Re^{J}\left(\mathrm{e}^{-i \theta} A\right)\right]=$ 0 . Thus $[C, H]=[C, K]=0$, which implies that $H, K$ can be expressed as polynomials in $C$ and so $A$ is $J$-normal.

Corollary 2.3. Let $J, C$ be under the conditions of Theorem 2.2 and let $A \in M_{n}$ be non $J$-normal. Then $W_{C}^{J}(A)$ is a $C^{(1)}$-regular curve.

Proof. Since $W_{C}^{J}(A)$ is convex, the nondifferentiable points of its boundary are corners. If there exists at least one corner, by Theorem 2.2, $A$ is $J$-normal, a contradiction.

By Tarski-Seidenberg theorem [7], the boundary of $W_{C}^{J}(A)$ lies in an algebraic curve. An exact algorithm to provide the equation of an algebraic curve containing $\partial W_{C}^{J}(A)$ is given by a similar method to the one in [2] due to Chien and Nakazato.

Example 2.1. Let $J=I_{2} \oplus-I_{1}, C=\operatorname{diag}(1,0,-1)$ and let

$$
A=\left(\begin{array}{rrr}
2 & i & 0 \\
i & 1 & i \\
0 & -i & -2
\end{array}\right) \text {. }
$$

The boundary of $W_{C}^{J}(A)$ lies in an algebraic curve $L(X, Y)=0$ of order 16 . The polynomial $L(X, Y)$ is given by

$$
\begin{aligned}
& L(X, Y)=-19683 X^{12} Y^{4}+76545 X^{10} Y^{6}-79542 X^{8} Y^{8}-8155 X^{6} Y^{10} \\
&+26514 X^{4} Y^{12}+8505 X^{2} Y^{14}+729 Y^{16}+1250964 X^{10} Y^{4}-2579850 X^{8} Y^{6} \\
&+2632812 X^{6} Y^{8}-2244970 X^{4} Y^{10}-315900 X^{2} Y^{12}+73710 Y^{14} \\
&-30362202 X^{8} Y^{4}+28179060 X^{6} Y^{6}-2850861 X^{4} Y^{8}+8523515 X^{2} Y^{10} \\
&+1233531 Y^{12}-186624 X^{10}+786240 X^{8} Y^{2}+344964500 X^{6} Y^{4} \\
&-101340750 X^{4} Y^{6}-138483138 X^{2} Y^{8}-32015970 Y^{10}+11321856 X^{8} \\
&-25683840 X^{6} Y^{2}-1775064187 X^{4} Y^{4}-98335965 X^{2} Y^{6}+131658345 Y^{8} \\
&-255820032 X^{6}+269156160 X^{4} Y^{2}+3366850448 X^{2} Y^{4}+323668800 Y^{6} \\
&+2578065408 X^{4}-920666880 X^{2} Y^{2}-2084332032 Y^{4}-10407481344 X^{2} \\
&-603832320 Y^{2}+8074100736 .
\end{aligned}
$$


We briefly describe how this polynomial is obtained. The curve $L(X, Y)=0$ is defined as the dual curve of an algebraic curve $M(1, x, y)=0$ of order 6. A generic point $\left(X_{0}, Y_{0}\right)$ of the curve $L(X, Y)=0$ corresponds to the tangent $X_{0} x+Y_{0} y+1=0$ of the curve $M(1, x, y)=0$ at a non-singular point $\left(x_{0}, y_{0}\right)$ of the curve $M(1, x, y)=0$. By this relation we can obtain the polynomial $L(X, Y)$ from the homogeneous polynomial $M(t, x, y)$. The polynomial $M(t, x, y)$ is defined as

$$
\begin{aligned}
& M(t, x, y)= \\
& \quad=\left[t-\left(\phi_{1}(x, y)-\phi_{2}(x, y)\right)\right]\left[t-\left(\phi_{1}(x, y)-\phi_{3}(x, y)\right)\right]\left[t-\left(\phi_{2}(x, y)-\phi_{1}(x, y)\right)\right] \\
& \quad \times\left[t-\left(\phi_{2}(x, y)-\phi_{3}(x, y)\right)\right]\left[t-\left(\phi_{3}(x, y)-\phi_{1}(x, y)\right)\right]\left[t-\left(\phi_{3}(x, y)-\phi_{2}(x, y)\right)\right]
\end{aligned}
$$

where $\phi_{j}(x, y)(j=1,2,3)$ satisfy the equation

$$
\begin{aligned}
\operatorname{det}\left(t I_{3}+x H+y K\right) & =t^{3}+t^{2} x-4 t x^{2}-4 x^{3}+4 x y^{2} \\
& =\left(t-\phi_{1}(x, y)\right)\left(t-\phi_{2}(x, y)\right)\left(t-\phi_{3}(x, y)\right)
\end{aligned}
$$

for every $(x, y) \in \mathbb{C}^{2}$.

The polynomial $M(t, x, y)$ has integral coefficients and $L(X, Y)$ is obtained as a factor of the resultant $L_{0}(X, Y)$ of the polynomial

$$
Y^{6} M(1, x,-1 / Y-x X / Y)=M(Y, x Y,-1-x X)
$$

and its derivative with respect to $x$. By factorizing $L_{0}$ in the polynomial ring $\mathbb{Z}[t, x, y]$, we can obtain the polynomial $L(t, x, y)$. Popular computer softwares for symbolic calculation, e.g., "Mathematica", "Maple" , etc, are available to perform such a factorization. In this example we find

$$
M(t, x, y)=t^{6}-26 t^{4} x^{2}+169 t^{2} x^{4}-144 x^{6}-560 x^{4} y^{2}+432 x^{2} y^{4} .
$$

\section{With flat portions in mind}

We recall that a supporting line of a convex set $S \subset \mathbb{C}$ is a line containing a boundary point of $S$, throughout denoted by $\partial S$, and defining two half planes, such that one of them does not contain $S$. Suppose that the straight line

$$
\ell=\left\{(x, y) \in \mathbb{R}^{2}: a x+b y+c=0, a, b, c \in \mathbb{R}\right\}
$$

is a supporting line of $W_{C}^{J}(A)$. If $\ell \cap \partial W_{C}^{J}(A)$ contains more than one point, then $\ell \cap \partial W_{C}^{J}(A)$ is called a flat portion on the boundary of $W_{C}^{J}(A)$. If $A$ is $J$-normal, then $\partial W_{C}^{J}(A)$ is a union of flat portions as shown in Theorem 3.2. 
Theorem 3.1. Let $A=H+i K$, where $H=\Re^{J}(A)$ and $K=\Im^{J}(A)$ are $J$-Hermitian and $H$ is $J$-unitarily diagonalizable with non interlacing eigenvalues. Let $\lambda_{1}^{\prime}>\cdots>\lambda_{s}^{\prime}$ be the $s$ distinct eigenvalues of $H$ with respective multiplicities $n_{1}, \cdots, n_{s}$. Let $C=C_{1} \oplus \cdots \oplus C_{s}, J=J_{1} \oplus \cdots \oplus J_{s}$, and

$$
K=\left(\begin{array}{cccc}
K_{11} & K_{12} & \cdots & K_{1 s} \\
K_{21} & K_{22} & \cdots & K_{2 s} \\
\cdots & \cdots & \cdots & \cdots \\
K_{s 1} & K_{s 2} & \cdots & K_{s s}
\end{array}\right)
$$

where $C_{j}, J_{j}, K_{j j} \in M_{n_{j}}, j=1, \cdots$, s. If at least one of the sets $W_{C_{t}}^{J_{t}}\left(K_{t t}\right)$ does not reduce to a singleton, then $\partial W_{C}^{J}(A)$ has a flat portion parallel to the imaginary axis.

Proof. Let $H=\operatorname{diag}\left(\lambda_{1}, \cdots, \lambda_{n}\right)$ with $\lambda_{1} \geq \cdots \geq \lambda_{r}>\lambda_{r+1} \geq \cdots \geq \lambda_{n}$, and $C=\operatorname{diag}\left(c_{1}, \cdots, c_{n}\right)$ with $c_{r+1} \geq \cdots \geq c_{n}>c_{1} \geq \cdots \geq c_{r}$. So there exists a line parallel to the imaginary axis separating $\sigma_{+}^{J}(A)$ and $\sigma_{-}^{J}(A)$. Then by Theorem 1.1 of [1]

$$
\left\{\operatorname{tr}\left(C U H U^{-1}\right): U \in \mathcal{U}_{r, n-r}\right\}=\left(-\infty, a_{0}\right], \quad a_{0}=\sum_{j=1}^{n} c_{j} \lambda_{j} .
$$

We show that

$$
\begin{gathered}
\left\{\operatorname{tr}\left(C U K U^{-1}\right): \operatorname{tr}\left(C U H U^{-1}\right)=a_{0}, U \in \mathcal{U}_{r, n-r}\right\} \\
=W_{C_{1}}^{J_{1}}\left(K_{11}\right)+W_{C_{2}}^{J_{2}}\left(K_{22}\right)+\cdots+W_{C_{s}}^{J_{s}}\left(K_{s s}\right) .
\end{gathered}
$$

In fact, if $\operatorname{tr}\left(C U H U^{-1}\right)=a_{0}$, then there exist $V, W \in \mathcal{U}_{r, n-r}$ such that $U=W V$ and $W C W^{-1}=C, V H V^{-1}=H$. Without loss of generality we may assume that $H=\left(\lambda_{1}^{\prime} I_{n_{1}}\right) \oplus \cdots \oplus\left(\lambda_{s}^{\prime} I_{n_{s}}\right)$. Therefore, $V=V_{1} \oplus \cdots \oplus V_{s}$, where $V_{j}$ has size $n_{j}$ and $V_{j} J_{j} V_{j}^{*}=J_{j}$, so that

$$
V K V^{-1}=\left(\begin{array}{ccc}
V_{1} K_{11} V_{1}^{-1} & \cdots & V_{1} K_{1 s} V_{s}^{-1} \\
\vdots & \ddots & \vdots \\
V_{s} K_{s 1} V_{1}^{-1} & \cdots & V_{s} K_{s s} V_{s}^{-1}
\end{array}\right)
$$

and

$$
\operatorname{tr}\left(C U K U^{-1}\right)=\sum_{j=1}^{s} \operatorname{tr}\left(C_{j} V_{j} K_{j j} V_{j}^{-1}\right)
$$


Let $\ell$ be the supporting line of $W_{C}^{J}(A)$ paralell to the imaginary axis. Thus

$$
\ell \cap \partial W_{C}^{J}(A)=\left\{a_{0}+i \sum_{j=1}^{s} \operatorname{tr}\left(C_{j} V_{j} K_{j j} V_{j}^{-1}\right): V_{j} J_{j} V_{j}^{*}=J_{j}\right\} .
$$

This intersection is a singleton if and only if each summand of the above Minkowski sum is a singleton, namely $\sum_{j=1}^{n} c_{j} \alpha_{j}$. If in the above sum there exists at least one set $W_{C_{t}}^{J_{t}}\left(K_{t t}\right)$ which is not a singleton, then $\ell \cap \partial W_{C}^{J}(A)$ is a line segment, a half-line or the whole line according to the following. If $n_{1}+\cdots+n_{t}=r$, then, for some $k, W_{C_{k}}^{J_{k}}\left(K_{k k}\right)=W_{C_{k}}\left(K_{k k}\right)$ is a line segment and $\ell \cap \partial W_{C}^{J}(A)$ is also a line segment. If $n_{1}+\cdots+n_{t}<r<n_{1}+\cdots+n_{t+1}$ and $W_{C_{t+1}}^{J_{t+1}}\left(K_{t+1 t+1}\right)$ is a singleton, then $\ell \cap \partial W_{C}^{J}(A)$ is still a line segment. Otherwise, this intersection is a half-line of $\ell$ or the whole line $\ell$. The result follows.

Let $C=\operatorname{diag}\left(c_{1}, \cdots, c_{n}\right)$ with $c_{r+1} \geq \cdots \geq c_{n}>c_{1} \geq \cdots \geq c_{r}$. We also assume that $A=\operatorname{diag}\left(\alpha_{1}, \cdots, \alpha_{n}\right), \sigma_{+}^{J}(A)=\left\{\alpha_{1}, \cdots, \alpha_{r}\right\}$ and $\sigma_{-}^{J}(A)=$ $\left\{\alpha_{r+1}, \cdots, \alpha_{n}\right\}$. Let $S_{n}$ denote the symmetric group of degree $n$ and let $S_{r, n-r}$ be the set of permutations of the form $\sigma=\sigma_{1} \circ \sigma_{2}$, where $\sigma_{1}, \sigma_{2} \in S_{n}$ are such that $\sigma_{1}(j)=j, j \in\{r+1, \cdots, n\}$ and $\sigma_{2}(j)=j, j \in\{1, \cdots, r\}$. The $r !(n-r)$ ! points

$$
z_{\sigma}=\sum_{j=1}^{n} c_{j} \alpha_{\sigma(j)}, \quad \sigma \in S_{r, n-r}
$$

belong to $W_{C}^{J}(A)$ and are the so-called $\sigma$-points.

For a fixed $\sigma \in S_{r, n-r}$ and $k, l \in\{1, \cdots r\}$ or $k, l \in\{r+1, \cdots n\}$, the $\frac{r(r-1)}{2}+\frac{(n-r)(n-r-1)}{2}$ line segments

$$
\ell_{k, l}^{\sigma}=\left\{\sum_{j=1}^{n} c_{j} \alpha_{\sigma(j)}-t\left(\alpha_{\sigma(k)}-\alpha_{\sigma(l)}\right)\left(c_{k}-c_{l}\right): 0 \leq t \leq 1\right\}
$$

are contained in $W_{C}^{J}(A)$. The line segment $\ell_{k, l}^{\sigma}$ is generated by the $J$-unitary matrices obtained from the identity replacing the entries $(k, k),(k, l),(l, k)$, $(l, l)$ respectively by $\cos \theta,-\sin \theta, \sin \theta, \cos \theta$. 
For a fixed $\sigma \in S_{r, n-r}$ and $l \in\{1, \cdots r\}, k \in\{r+1, \cdots n\}$, the $r(n-r)$ half-lines

$$
\mathcal{L}_{k, l}^{\sigma}=\left\{\sum_{j=1}^{n} c_{j} \alpha_{\sigma(j)}+t\left(\alpha_{k}-\alpha_{l}\right): 0 \leq t<\infty\right\}
$$

are contained in $W_{C}^{J}(A)$. The ray $\mathcal{L}_{k, l}^{\sigma}$ is generated by the $J$-unitary matrices obtained from the identity replacing the entries $(k, k),(k, l),(l, k),(l, l)$ respectively by $\cosh \theta, \sinh \theta, \sinh \theta, \cosh \theta$.

Theorem 3.2. Let $J=I_{r} \oplus-I_{n-r}$ and let $A=\operatorname{diag}\left(\alpha_{1}, \cdots, \alpha_{n}\right)$ be a $J$-normal matrix such that there exists a line separating $\sigma_{+}^{J}(A)=\left\{\alpha_{1}, \cdots, \alpha_{r}\right\}$ and $\sigma_{-}^{J}(A)=\left\{\alpha_{r+1}, \cdots, \alpha_{n}\right\}$. Let $C=\operatorname{diag}\left(c_{1}, \cdots, c_{n}\right)$ with $c_{r+1} \geq \cdots \geq$ $c_{n}>c_{1} \geq \cdots \geq c_{r}$. Then

$$
W_{C}^{J}(A)=\operatorname{Co}\left\{\mathcal{L}_{k, l}^{\sigma}: \sigma \in \mathcal{S}_{r, n-r}, l=1, \ldots, r, k=r+1, \ldots, n\right\},
$$

where Co $\{\cdot\}$ denotes the convex hull of $\{\cdot\}$.

Proof. Since $W_{C}^{J}(A)$ is convex, then

$$
\text { Co }\left\{\mathcal{L}_{k, l}^{\sigma}: \sigma \in \mathcal{S}_{r, n-r}, l=1, \ldots, r, k=r+1, \ldots, n\right\} \subseteq W_{C}^{J}(A) .
$$

Next we show that the reversed inclusion also holds. Let $z$ be an arbitrary point in $\partial W_{C}^{J}(A)$. Then $z$ is either a corner or a regular point. If $z$ is a corner, then it is a $\sigma$-point. If $z$ is a regular point there exists a unique supporting line to $W_{C}^{J}(A)$ passing through it. Let $A=\mathrm{e}^{-i \theta}\left(H_{\theta}+i K_{\theta}\right)$, and so $H_{\theta}$ and $K_{\theta}$ commute due to the $J$-normality of $A$. Assume that $H_{\theta}$ has $s$ distinct eigenvalues, $\lambda_{1}^{\prime}=\lambda_{1}, \lambda_{2}^{\prime}=\lambda_{n_{1}+1}, \cdots, \lambda_{s}^{\prime}=\lambda_{n_{1}+\cdots+n_{s-1}+1}$ with multiplicities $n_{1}, \cdots, n_{s}$, respectively. Then $H_{\theta}$ and $K_{\theta}$ are simultaneously $J$-unitarily diagonalizable, being $n_{1}, \cdots, n_{s}$ the sizes of the diagonal blocks. Analogously to Theorem 3.1, we may conclude that

$$
\ell_{\theta} \cap \partial W_{C}^{J}(A)=\left\{a_{0}+i \sum_{j=1}^{s} \operatorname{tr}\left(C_{j} V_{j} K_{j j} V_{j}^{-1}\right): V_{j} J_{j} V_{j}^{*}=J_{j}\right\},
$$

where $\ell_{\theta}$ is the supporting line perpendicular to the direction $\arg (z)=-\theta$. This intersection is a singleton if and only if each summand of the above Minkowski sum is a singleton and reduces to $\sum_{j=1}^{n} c_{j} \alpha_{\sigma(j)}$ for a certain $\sigma \in S_{r, n-r}$. If in the above sum there exists at least one set $W_{C_{t}}^{J_{t}}\left(K_{t t}\right)$ which does not reduce to a singleton, then $\ell_{\theta} \cap \partial W_{C}^{J}(A)$ is a line segment, a half-line or the whole line. 
Remark 3.1. According to Theorem 3.2, $\partial W_{C}^{J}(A)$ is a convex polygon if $A$ is $J$-normal. Next we present an example of a non $J$-normal $A$ for which $\partial W_{C}^{J}(A)$ is still a convex polygon. Consider $J=\operatorname{diag}(1,1,1,1,-1)$, $C=\operatorname{diag}(1,0,0,0,0)$ and

$$
A=\left(\begin{array}{ccccc}
1+i & 0 & 0 & 0 & 0 \\
0 & 1-i & 0 & 0 & 0 \\
0 & 0 & 2 & b & 0 \\
0 & 0 & 0 & 2 & 0 \\
0 & 0 & 0 & 0 & -1
\end{array}\right)
$$

with $b \in \mathbb{R}$. We have

$$
\Re^{J}\left(\mathrm{e}^{-i \theta} A\right)=\left(\begin{array}{ccccc}
\cos \theta+\sin \theta & 0 & 0 & 0 & 0 \\
0 & \cos \theta-\sin \theta & 0 & 0 & 0 \\
0 & 0 & 2 \cos \theta & \frac{b}{2} \mathrm{e}^{-i \theta} & 0 \\
0 & 0 & \frac{b}{2} \mathrm{e}^{i \theta} & 2 \cos \theta & 0 \\
0 & 0 & 0 & 0 & -\cos \theta
\end{array}\right),
$$

with

$$
\begin{gathered}
\sigma_{+}^{J}\left(\Re^{J}\left(\mathrm{e}^{-i \theta} A\right)\right)=\left\{2 \cos \theta+\frac{b}{2}, 2 \cos \theta-\frac{b}{2}, \cos \theta+\sin \theta, \cos \theta-\sin \theta\right\} \\
\sigma_{-}^{J}\left(\Re^{J}\left(\mathrm{e}^{-i \theta} A\right)\right)=\{-\cos \theta\} .
\end{gathered}
$$

If $b \leq 2$ and $-2 \leq \tan \theta \leq 0$, the smallest eigenvalue in $\sigma_{+}^{J}\left(\Re^{J}\left(\mathrm{e}^{-i \theta} A\right)\right)$ is $\cos \theta-\sin \theta$, so that $1-i$ is a corner of $\partial W_{C}^{J}(A)$. If $b \leq 2$ and $0 \leq \tan \theta \leq 2$, the smallest eigenvalue in $\sigma_{+}^{J}\left(\Re^{J}\left(\mathrm{e}^{-i \theta} A\right)\right)$ is $\cos \theta+\sin \theta$, so that $1+i$ is a corner of $\partial W_{C}^{J}(A)$. Then, it follows trivially from Theorem 3.1 that $W_{C}^{J}\left(\Re^{J}\left(\mathrm{e}^{-i \theta} A\right)\right)=[1+i, 1-i] \cup\{1+i+(2+i) t: t \geq 0\} \cup\{1-i+(2-i) t: t \geq 0\}$. If $b=3$ and $-\pi / 3 \leq \theta \leq \pi / 3$, the smallest eigenvalue in $\sigma_{+}^{J}\left(\Re^{J}\left(\mathrm{e}^{-i \theta} A\right)\right)$ is $2 \cos \theta-b / 2$. Then, easy calculations based on Theorem 3.1 show that

$$
\begin{aligned}
& W_{C}^{J}\left(\Re^{J}\left(\mathrm{e}^{-i \theta} A\right)\right)=\left\{2+\frac{3}{2} \mathrm{e}^{i \phi}: \frac{2 \pi}{3} \leq \phi \leq \frac{4 \pi}{3}\right\} \\
& \cup\left\{\frac{5+i 3 \sqrt{3}}{4}+(1+i \sqrt{3}) t: t \geq 0\right\} \cup\left\{\frac{5-i 3 \sqrt{3}}{4}+(1-i \sqrt{3}) t: t \geq 0\right\} .
\end{aligned}
$$

In this case there are no corners in $\partial W_{C}^{J}(A)$. For $b=6, W_{C}^{J}(A)$ is the right half plane defined by $x=1$. For $b>6, W_{C}^{J}(A)$ is the whole complex plane. 
Theorem 3.3. Let $J, C$ and $A$ be under the conditions of the Theorem 3.2. If $z \in \partial W_{C}^{J}(A)$, then one of the following assertions holds:

(i) $z$ is a $\sigma$-point;

(ii) $z \in\left[z_{\sigma^{\prime}}, z_{\sigma^{\prime \prime}}\right]$, where $\sigma^{\prime}, \sigma^{\prime \prime} \in S_{r, n-r}$ differ by a transposition;

(iii) $z \in\left\{z_{\sigma}+t\left(\alpha_{k}-\alpha_{l}\right): t \geq 0\right\}$, for some $l \in\{1, \ldots, r\}, k \in\{r+1, \ldots, n\}$.

Proof. By Theorem 3.1, $W_{C}^{J}(A)$ is the closed convex-hull of the half-lines (8). If $z \in \partial W_{C}^{J}(A)$, having in mind that $\partial W_{C}^{J}(A)$ is a convex curve, then $z$ is a corner or a regular point. In the first case, $z$ is a $\sigma$-point. If $z$ is a regular point, let $\pi / 2-\theta$ be the slope of the supporting line passing through $z$. By a perturbation, we may suppose that the eigenvalues of $A$ define $\left(\begin{array}{l}n \\ 2\end{array}\right)$ distinct directions. Then $H_{\theta}$ has precisely one double eigenvalue and the intersection of that supporting line with $\partial W_{C}^{J}(A)$ is either a line segment, whose endpoints are $\sigma$-points which differ by a transposition, or a half-ray of the stated type.

Theorem 3.4. Let $A \in M_{n}$. If $\Re^{J}\left(\mathrm{e}^{-i \theta} A\right)$ has imaginary eigenvalues for any $\theta \in \mathbb{R}$, then $W_{C}^{J}(A)$ is the whole complex plane. If the spectrum of $\Re^{J}\left(\mathrm{e}^{-i \theta_{0}} A\right)$ is real for $\theta=\theta_{0}$ and $\Re^{J}\left(\mathrm{e}^{-i \theta} A\right)$ has imaginary eigenvalues for $\theta \neq \theta_{0}$, then $W_{C}^{J}(A)$ is either a half plane or the whole complex plane.

Proof. If for any $\theta \in \mathbb{R}$, the matrix $\Re^{J}\left(\mathrm{e}^{-i \theta} A\right)$ has imaginary eigenvalues, then the projection of $W_{C}^{J}(A)$ on the line with slope $\pi / 2+\theta$ is the whole line. If there exists a unique $\theta_{0} \in \mathbb{R}$, such that the spectrum of $\Re^{J}\left(\mathrm{e}^{-i \theta_{0}} A\right)$ is real, then the projection of $W_{C}^{J}(A)$ on the line with slope $\pi / 2+\theta_{0}$ is either the whole line or a half-line by Theorem 1.1 of [1].

\section{Numerical algorithm and Examples}

Based on Westwick's theorem, Li et al [5] provided an algorithm to plot $\partial W_{C}(A)$ for $C$ a Hermitian matrix. A numerical algorithm to draw the boundary of $W_{C}^{J}(A)$ for an arbitrary $A \in M_{n}$ and $C$ a $J$-Hermitian matrix is here presented. Theorem 2.1, Lemma 5.3 and Theorem 1.1 of [1] provide the general principle. Our approach uses the elementary idea that $\partial W_{C}^{J}(A)$ may be traced by computing the supporting lines of $W_{C}^{J}(A)$ as specified below. The accuracy is quite good. 


\section{Algorithm}

Step 1: For an arbitrary complex matrix $A \in M_{n}$, an involutive Hermitian matrix $J \in M_{n}$ and a real matrix $C=\operatorname{diag}\left(c_{1}, \ldots, c_{n}\right)$, compute the eigenvectors of $C, v_{j}, j=1, \ldots, n$ and construct two vectors formed by the eigenvalues of $C$ in $\sigma_{+}^{J}(C)$ and in $\sigma_{-}^{J}(C)$. If they do not interlace, go to Step 2. Otherwise, go to Step 8 and we have a degenerate case.

Step 2: Compute the eigenvalues of the matrix $H_{\theta_{k}}:=\Re^{J}\left(\mathrm{e}^{-i \theta_{k}} A\right)$, with

$$
\theta_{k}=\frac{\pi(k-1)}{2 m}
$$

for some positive integer $m$ and for $k=1, \ldots, 2 m$. Construct the vector formed by all the values of $k$ such the matrix $H_{\theta_{k}}$ has real spectrum with anisotropic eigenvectors. If this vector is nonempty, go to Step 3. Otherwise, go to Step 8 and we have a degenerate case.

Step 3: For each $\theta_{k}$ described above, compute the eigenvalues of the matrix $H_{\theta_{k}}$, and the associated eigenvectors $u_{j}(k), j=1, \ldots, n$. Construct two vectors formed by the eigenvalues of $H_{\theta_{k}}$ in $\sigma_{+}^{J}\left(H_{\theta_{k}}\right)$ and in $\sigma_{-}^{J}\left(H_{\theta_{k}}\right)$.

Step 4: Investigate the existence of directions for which the eigenvalues of the matrix $H_{\theta_{k}}$ do not interlace. If these directions exist and are not all the directions given by (9), go to Step 5. Otherwise, follow to Step 8.

Step 5: Let $r$ and $n-r$ be the number of eigenvalues of $C$ in $\sigma_{+}^{J}(C)$ and $\sigma_{-}^{J}(C)$. For each $k=1, \ldots, 2 m$, compute the $r !(n-r)$ ! points

$$
z_{\sigma}\left(\theta_{k}\right)=\sum_{s=1}^{n} c_{s} \lambda_{\sigma}\left(H_{\theta_{k}}\right),
$$

where $\sigma=\sigma_{1} \circ \sigma_{2} \in S_{r, n-r}$ and $\lambda_{1}, \ldots, \lambda_{n}$ are the eigenvalues of $H_{\theta_{k}}$.

Step 6: Since the eigenvalues of $C$, as well as those of each $H_{\theta_{k}}$, do not interlace, we can have one of the two relations given in (4). So, if the relation (4) is the same for the eigenvalues of the matrices $C$ and $H_{\theta_{k}}$, we put $\lambda(k):=$ $\min z_{\sigma}\left(\theta_{k}\right)$. Otherwise, $\lambda(k):=\max z_{\sigma}\left(\theta_{k}\right)$.

Step 7: For each $k=1, \ldots, 2 m$, plot the supporting line of $W_{C}^{J}(A)$ defined by:

$$
x \cos \left(\theta_{k}\right)-y \sin \left(\theta_{k}\right)=\lambda(k) .
$$


Step 8: (Degenerate cases) Compute

$$
\sum_{s=1}^{n} c_{s} \frac{\zeta_{j_{s}}^{*} J A \zeta_{j_{s}}}{\zeta_{j_{s}}^{*} J \zeta_{j_{s}}},
$$

for a sample of anisotropic vectors $\zeta_{j}=\left(\zeta_{j_{1}}, \ldots, \zeta_{j_{n}}\right)$ randomly chosen. The distribution of these points allows to conclude whether $W_{C}^{J}(A)$ is the complex plane (possibly an half-plane) or a line (possibly an half-line).

Remark 4.1. Obviously, if $J=I,-I$, then $W_{C}^{J}(A)$ reduces to the $C$-numerical range and our algorithm and program also work in this case. Its speed and accuracy are equivalent to those of the program in [5].

We illustrate the algorithm with the following examples. All the figures presented in this note were generated by our Matlab program available at the following website: http://www.mat.uc.pt/ bebiano.

Example 4.1. Let $J=I_{2} \oplus-I_{2}, C=\operatorname{diag}(2,1,-1,-2)$ and let

$$
A=\left(\begin{array}{rrrr}
2 & i & 0 & 0 \\
i & 1 & i & 0 \\
0 & -i & -1 & -i \\
0 & 0 & -i & -2
\end{array}\right) .
$$

$W_{C}^{J}(A)$ is represented in Figure 1 by the supporting lines.

Example 4.2. Let $J=I_{2} \oplus-I_{2}, C=\operatorname{diag}(2,1,-1,-2)$ and let

$$
A=\left(\begin{array}{rrrr}
2 & i & 0 & 0 \\
i & 2 & i & 0 \\
0 & -i & -1 & -i \\
0 & 0 & -i & -2
\end{array}\right)
$$

$W_{C}^{J}(A)$ is represented in Figure 2 by the supporting lines. The line segment $[10+i, 10-i]$ is contained in the boundary.

Example 4.3. Let $J=I_{2} \oplus-I_{2}, C=\operatorname{diag}(2,1,-1,-2)$ and

$$
A=\operatorname{diag}(3+2 i, 1-i,-1+i,-3-2 i) \text {. }
$$

In this case,

$\partial W_{C}^{J}(A)=\{10+(1-i) t: t \geq 0\} \cup[10,14+6 i] \cup\{14+6 i+(3+2 i) t: t \geq 0\}$, and $W_{C}^{J}(A)$ is represented in Figure 3 by the supporting lines. 


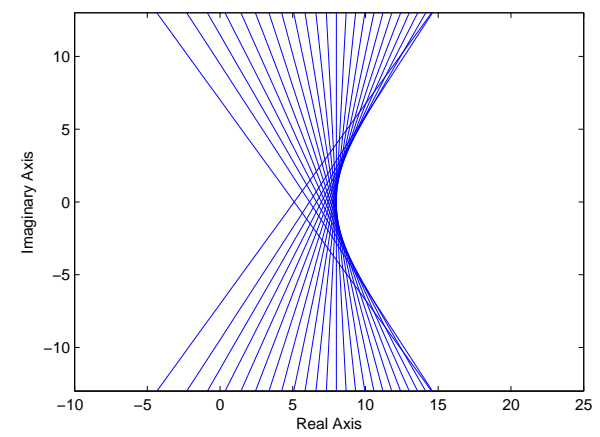

Figure 1. Supporting lines of $W_{C}^{J}(A)$, for $J, C$ and $A$ as in Example 4.1.

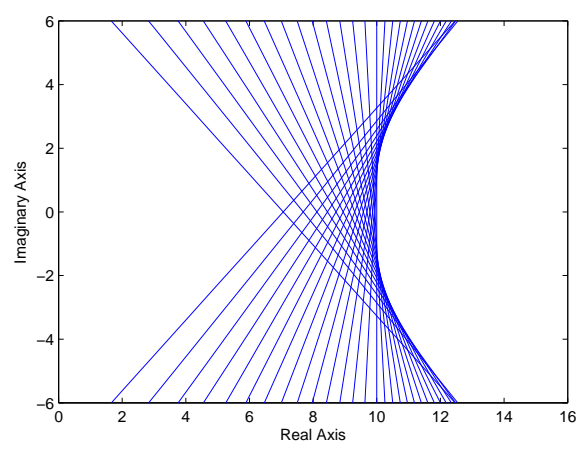

Figure 2. Supporting lines of $W_{C}^{J}(A)$, for $J, C$ and $A$ as in Example 4.2.

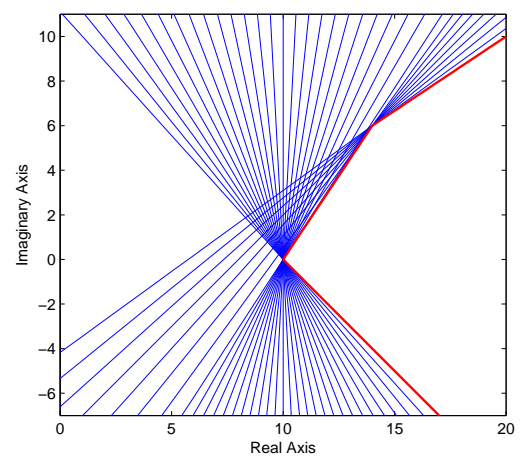

Figure 3. Supporting lines of $W_{C}^{J}(A)$, for $J, C$ and $A$ as in Example 4.3. 


\section{References}

[1] N. Bebiano, H. Nakazato, J. da Providência, R. Lemos, G. Soares, Inequalities for J-Hermitian matrices, Linear Algebra and its Applications 407 (2005), 125-139.

[2] M-T. Chien and H. Nakazato, Boundary generating curves of the $c$-numerical range, Linear Algebra and its Applications 294 (1999), 67-84.

[3] F. Hausdorff, Das Wertevorrat einer Bilinear form, Math. Zeit. 3 (1919), 314-316.

[4] N. Jacobson, Basic algebra I, W.H. Freeman \& Co, (1985) 2nd Ed.

[5] C.-K. Li, C.H. Sung and N.K. Tsing, $C$-convex matrices: Characterizations, inclusion relations and normality, Linear and Multilinear Algebra 25 (1989), 275-287.

[6] C.-K. Li and L. Rodman, Remarks on numerical ranges of operators in spaces with an indefinite metric, Proc. Amer. Math. Soc. 126 (1998), 973-982.

[7] H. Nakazato, N. Bebiano and J. da Providência, Convexity of the Krein space tracial numerical range and Morse theory, Mathematical Reports of the Academy of Science, Royal Society of Canada, in press.

[8] Y.T. Poon, Another proof of a result of Westwick, Linear and Multilinear Algebra 9 (1980), $35-37$.

[9] T.Y. Tam, Convexity of generalized numerical range associated with a compact Lie group, $J$. Aust. Math. Soc. 72 (2002), 57-66.

[10] R. Westwick, A theorem on numerical range, Linear and Multilinear Algebra 2 (1975), 311-315.

N. Bebiano

CMUC, Department of Mathematics, University of Coimbra, 3001-454 Coimbra, Portugal

E-mail address: bebiano@mat.uc.pt

H. NAKAZATO

Department of Mathematical Sciences, 036-8561 Hirosaki, Japan

E-mail address: nakahr@cc.hirosaki-u.ac.jp

A. NATA

CMUC and Polytechnic Institute of Tomar, Department of Mathematics, 2300-313 Tomar, PORTUGAL

E-mail address: anata@ipt.pt

J. DA PRovidÊNCIA

Department of Physics, University of Coimbra, 3004-516 Coimbra, Portugal

E-mail address: providencia@teor.fis.uc.pt 